Supporting Notes

to

\title{
Ethanol's Energy Return on Investment: A Survey of the Literature 1990-Present
}

\author{
Roel Hammerschlag \\ Institute for Lifecycle Environmental Assessment \\ PO Box 22437 \\ Seattle, WA 98122 \\ tel. 206-406-7792 \\ roel@ilea.org
}

January, 2006 


\section{Magnitude of error generated by the conversion from LHV to HHV}

The conversion was performed simply by multiplying all reported LHV energy values by 1.09 , the ratio between HHV and LHV of ethanol, and coincidentally the ratio between HHV and LHV of U.S. average fossil fuel consumption. This will introduce a minor and nonsystematic error into our recalculated values of $r_{E}$, since the HHV to LHV ratios of the specific fuels assumed by the authors will vary both above and below 1.09 by at most a few percent. An exact conversion was impossible because no authors reported exact fuel mixes for each process step. The introduced error is probably about one order of magnitude smaller than the gross correction to each study's energy inputs.

\section{ISO-preferred allocation methodology followed by Kim \& Dale 2005}

The preferred approach is system expansion, in which the energy analysis is expanded to include the production chains associated with the entire market for coproducts, thus avoiding allocation altogether. The system expansion approach can infer the proportion of the ethanol plant's energy that is dedicated to the ethanol, and Kim \& Dale take the liberty to do this, thus allowing the coproduct energy input of $4.8 \mathrm{MJ} / \mathrm{L}$ we report in Table 1.

All of the other authors follow the replacement approach: the energy inputs assigned to non-ethanol coproducts is equal to the energy required to manufacture the same coproducts in a dedicated, independent process. Though the replacement approach is technically reasonable, and allowed under the ISO standard, it is also the source of the misleading concept of an "energy credit," because it results in a specific quantity of energy to subtract from the ethanol plant's gross output.

\section{Remarks on conservative assumptions to corn ethanol data reported by Pimentel \& Patzek 2005}

The manufacturing energy for nitrogen fertilizer is stated by Pimentel \& Patzek as $66 \mathrm{MJ} / \mathrm{kg}$, which is equivalent to 1960-vintage processes per a source document by Patzek (Patzek, T. W. Thermodynamics of the Corn-Ethanol Biofuel Cycle. Critical Reviews in Plant Science 2004.) Modern processes consume only about $39 \mathrm{MJ} / \mathrm{kg}$, according to the same document.

The energy value associated with seed production is stated to be about 2,200 MJ/ha and is referenced to a 1980 resource (Pimentel, D; Handbook of Energy Utilization in Agriculture; CRC Press: Boca Raton, FL, 1980.) In contrast, Gabroski states approximately $220 \mathrm{MJ} / \mathrm{ha}$ and Shapouri et al state $77 \mathrm{MJ} / \mathrm{ha}$, both an order of magnitude lower than Pimentel \& Patzek. 
Pimentel \& Patzek state metered electricity use at the plant to be $0.39 \mathrm{kWh} / \mathrm{L}$. The citation provided for this is http://www.ilcorn.org/Ethanol/Ethan_Studies/Ethan_Energy_Bal/ethan_energy_bal.html, a web page hosted by the Illinois Cōrn Growers' Association. Shapouri et al, reporting a more typical $0.29 \mathrm{kWh} / \mathrm{L}$, cite a telephone survey of 17 dry mills conducted by BBI International in 2001. Pimentel \& Patzek also use a very high electricity heat rate of 3.3 (on an HHV basis), escalating the already high metered electricity use to an even greater primary energy requirement. They do not mention the distinction between nonrenewable and renewable energy in their paper, so the high electricity heat rate may reflect inclusion of hydroelectric and nuclear power in $\mathrm{E}_{\mathrm{in}, \text { nonrenewable. }}$

Kim \& Dale's allocation procedure is the most sophisticated, and is thoroughly documented in a separate article (Kim, S.; Dale, B. Allocation Procedure in Ethanol Production System From Corn Grain. Int. J. LCA 2002, 7 (4) 237-43.) They allocate approximately $25 \%$ of the dry mill's energy consumption to the coproduct. This provides further evidence that the $7 \%$ used by Pimentel \& Patzek is probably unreasonably low.

\section{Valuing the surplus electricity in $E_{\text {out }}$ for cellulosic ethanol}

Some analysts have argued that the surplus electricity should be multiplied by a heat rate before being added to $E_{\text {out }}$. Doing so requires choosing a uniform heat rate, or

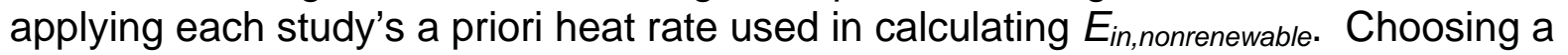
uniform heat rate would unnecessarily add an assumption (the chosen heat rate) to an otherwise objective literature survey. Applying each study's a priori heat rate implies a similar assumption: namely that the heat rate of electricity displaced by the surplus electricity is equal to the heat rate assumed by the authors for input electricity. Applying heat rates either way would likely be perceived as advocacy for cellulosic ethanol, since it increases the values of $r_{E}$.

Multiplying the surplus electricity by a grid electricity heat rate also raises a methodological question, because the utility of the primary fuel displaced according to a grid heat rate is much higher than the utility of the surplus electricity. Primary fuel can be stored and dispatched at will to generate electricity at a grid-connected generator; surplus electricity associated with ethanol manufacture is not dispatchable, is generated in relatively small quantities, and may not be at a location convenient to the grid. A grid heat rate overestimates the value of the surplus electricity as a product in the energy economy.

\section{Physical interpretation of the $r_{E}$ calculated for cellulosic ethanol}

The $r_{E}$ reported in the paper do not represent the cellulosic ethanol energy return on fossil energy investment; rather they represent total energy return on fossil energy investment associated with cellulosic ethanol. The numeric difference is as subtle as the linguistic one; indeed, if ethanol and electricity are assigned allocation factors 
proportional to their commodity energy values, then the resulting, strict ethanol $\mathrm{r}_{\mathrm{E}}$ are identical to the total energy $r_{E}$ reported in Table 2.

\section{World LNG reserves}

The largest reserves of natural gas are located in, in decreasing order, Russia, Iran, Qatar, Saudi Arabia, United Arab Emirates, U.S., Algeria, Venezuela, Nigeria, Iraq and Indonesia. CIA World Factbook; United States Central Intelligence Agency: Washington, DC, 2005. Available at http://www.cia.gov/cia/publications/factbook/rankorder/2179rank.html.

\section{The Brazilian experience}

Studies of the Brazilian ethanol industry frequently report $r_{E} \approx 10$ (e.g. de Carvalho Macedo, I.; Leal, M. R. L. V.; da Silva, J. E. A. R.; Assessment of Greenhouse Gas Emissions in the Production and Use of Fuel Ethanol in Brazil; Sao Paulo Secretariat of the Environment: 2004). The sugarcane ethanol produced in Brazil is a starch ethanol, but the industrial process is almost always powered by combustion of bagasse - the fibrous (lignin-containing) portion of sugarcane that resists fermentation. The result is that very little fossil fuel energy is needed to produce the ethanol. The equivalent process in the United States would be powering of corn ethanol plants by combustion of corn stover. 